\title{
Sprachgeographische Aspekte der Morphologie und Verschriftung in schweizerdeutschen Chats
}

\author{
Beat Siebenhaar (Bern)
}

\begin{abstract}
The regional chat-rooms in Switzerland show an extremely high portion of dialectal contributions (up to 90\%). This non-standardized spontaneous writing of a dialectal language still reflects the geolinguistic distribution described in the linguistic atlas of German speaking Switzerland SDS (1962-1997) based on recordings of the 1940s and 1950s.

This paper shows some reflexes of this geolinguistic distribution in four chat-rooms. The graphemic representation of the ending vowel of infinitives clearly confirms the traditional structure. Deviating e-graphemes in chat-rooms of alpine regions can be rated as common Swiss German variants for centralized vowels. On the other hand ä-graphemes in chat-rooms of the Swiss midlands are to be rated as marking of the phonetic deviation from the standard German pronunciation. This variation is not only found in inherited words, but also in neologisms with an almost identical distribution. The SDS illustrates a distribution for the use of t-endings in the 2nd and 3rd singular of sein 'to be'. These t-flexives cannot be found anymore in midland chat-rooms. They appear only in alpine chat-rooms, and there they become morphologized in a new way. The dialectal writing of neologisms confirms the validity of the principles for the Standard German writing.
\end{abstract}

\section{Schweizerdeutsch im Netz}

Wer im Netz deutschsprachige Seiten besucht, erwartet nicht, dass er Mundart findet, sondern die Schriftsprache, Standardsprache. Schließlich ist das die überregionale Sprachform und sie stellt auch in der Schweiz die unmarkierte Variante dar. Doch in diesem Bereich finden sich eine ganze Menge Web-Seiten, deren Gestalter sich der Welt in Mundart kundtun, wie in Tabelle 1 dargestellt. Neben rund 12'100 Seiten aus der Schweiz, ${ }^{1}$ die sowohl gehabt als auch gewesen als Hinweis auf standard-deutsche Seiten aufweisen, finden sich auch rund 3400, die mit verschiedenen Kombinationen der Schreibvarianten von ghaa und gsy erscheinen. Sie können damit als Seiten in einer schweizerdeutschen Mundart verstanden werden. Der Anteil mundartlicher Seiten auf dem .ch-Domain kann also auf rund $22 \%$ geschätzt werden.

\footnotetext{
${ }^{1}$ Domainnamen auf .ch
} 


\begin{tabular}{|l|l|l|l|l|l|l|l|l|}
\hline $\begin{array}{l}\text { gewesen }+ \\
\text { gehabt }\end{array}$ & gha + gsi & $\begin{array}{l}\text { ghaa }+ \\
\text { gsi }\end{array}$ & gha + gsii & $\begin{array}{l}\text { ghaa } \\
\text { gsii }\end{array}$ & gha + gsy & $\begin{array}{l}\text { ghaa }+ \\
\text { gsy }\end{array}$ & $\begin{array}{l}\text { gha }+ \\
\text { gsyy }\end{array}$ & $\begin{array}{l}\text { ghaa }+ \\
\text { gsyy }\end{array}$ \\
\hline $12^{\prime} 100$ & 2620 & 90 & 474 & 98 & 166 & 11 & 2 & 0 \\
\hline
\end{tabular}

Tabelle 1: Vorkommen von gehabt+gewesen / gha+gsi mit Schreibvarianten in Webseiten auf dem ch-Domain ${ }^{2}$

Wenn also schon eine ansehnliche Vertretung von Mundart auf Schweizer Webseiten zu beobachten ist, so ist im Bereich des Chats, der konzeptionell näher bei der Mündlichkeit steht, noch mehr Mundart zu erwarten. Aktuelle Arbeiten zum Chat in der Schweiz, wie Kelle (2000), Aschwanden (2001), Christen u. a. (i. Dr.) sowie Christen (eingereicht), belegen eine solche Verteilung. Zudem ist die Mundart auch gesamtdeutsch im Chat viel stärker vertreten als in übrigen schriftlichen Belegen (Christen, Tophinke, Ziegler unv.). In regional gebundenen Schweizer Chats nehmen die Dialekte eine geradezu dominierende Rolle ein, wie unten gezeigt werden soll. Dabei werden anders als in deutschen Chats, wo mit einzelnen isolierten Einheiten auf der Wortebene und mittels rituellen Begrüßungs- und Verabschiedungssequenzen regionale Identität vermittelt wird (Christen, Tophinke, Ziegler unv.), häufig oder sogar mehrheitlich ganze Turns mundartlich realisiert. Auf der Basis der Unterscheidung von konzeptioneller Mündlichkeit und konzeptioneller Schriftlichkeit nach Koch/Oesterreicher (1994) interpretiert Aschwanden (2001) diese vermehrte Verwendung der geschriebenen Mundart als eine Auflösung der seit rund 20 Jahren für die Deutschschweiz postulierten medialen Diglossie und als Hinwendung zu einer konzeptionellen Diglossie, in der die Mundart für Nähe, der Standard hingegen für Distanz verwendet wird. An dieser Stelle soll Aschwandens Position insofern relativiert werden, als die von ihr beschriebene Neustrukturierung der Diglossie nur den Nähebereich betrifft, wo auch in der schriftlichen Kommunikation vermehrt die Mundart verwendet wird. Im Distanzbereich gilt die mediale Diglossie weiterhin: die Standardsprache wird durch die verschriftete Mundart kaum in Frage gestellt. Der Dialekt dagegen ist auch dann die unmarkierte Varietät in der mündlichen Kommunikation, wenn die äußeren Bedingungen dem Distanzbereich zuzuordnen sind, also beispielsweise wenn sich die Kommunikationspartner fremd sind oder die Kommunikation öffentlich ist. Ausnahmen bilden fast nur diejenigen Kommunikationssituationen, in denen die Standardsprache institutionell festgelegt ist. ${ }^{3}$

Wenn nun die Mundart im Chat eine solch bestimmende Rolle einnimmt, so ist auf dem Hintergrund der starken dialektalen Diversität zu erwarten, dass sich diese Unterschiede auch in gechatteten Äußerungen finden. Die dialektale Gliederung der Deutschschweiz ist in den 1930er bis 1950er Jahren erhoben worden und im Sprachatlas der deutschen Schweiz (SDS 1962-1997) dokumentiert. Dass die dort festgehaltene Sprachstruktur in ihren wesentlichen Zügen immer noch gilt, hat Christen (1998) in Gesprächen mit Studierenden in überregionalen Fachhochschulen deutlich gezeigt. Dass sie auch in den Chats deutlich wird, wird im Folgenden gezeigt.

\footnotetext{
2 Google-Suche vom 10.10.2002. Doppelte Zählungen einzelner Webseiten sind nicht auszuschließen. Die Kombination gewesen + gehabt' + Varianten von 'gha' + 'gsi' ist beispielsweise 153 Mal vertreten, was auf die Verwendung beider Varietäten hindeutet.

${ }^{3}$ Diese wertvolle Präzisierung verdanke ich einem der beiden anonymen Gutachter.
} 

und Verschriftung in schweizerdeutschen Chats

In der Verschriftung von Dialekt liegt eine große Varianz, die in wesentlichen Teilen darauf zurückzuführen ist, dass es sich dabei um eine Schriftsprache ohne kodifizierte Norm handelt. Auch wenn im Lauf des 20. Jahrhunderts mehrere Versuche gemacht wurden, die Schreibung von Schweizer Mundarten zu vereinheitlichen - teilweise auf eine regionale (z. B. Marti 1972 für das Berndeutsche), teilweise auf überregionale eine Norm (Dieth 1938 für das Schweizerdeutsche) hin tendierend - (vgl. dazu Lötscher 1989, Lötscher 1990), so zeigen diese Normvorschläge im Chat reichlich wenig Niederschlag, wie unten gezeigt wird. ChatterInnen verwenden eine spontane Mundartschreibung, ohne sich an äußere Regeln zu halten.

\section{Datenbasis}

Um dies zu überprüfen, wurden vier als regional definierte Chats auf dem swisscom-Portal bluewin während drei langen Wochenenden aufgezeichnet. Es handelt sich um die Chaträume, die mit \#zuerich, \#bern, \#wallis und \#graubuenden überschrieben sind. Sie sind sowohl über ein Applet im Browser als auch über ein IRC-Programm ansprechbar. Die Aufnahmen erfolgten an drei aufeinander folgenden langen Wochenenden (25. Januar 2002 bis 11. Februar 2002). Für jeden Kanal wurden während 194 Stunden sämtliche Beiträge in eine Protokolldatei gesichert, daraus ergeben sich rund 455'218 Wörter in 122'441 Beiträgen, die für die Analyse genutzt werden können. Tabelle 2 gibt einen Überblick über die Grunddaten.

\begin{tabular}{|l|l|l|l|l|l|l|l|l|l|}
\hline $\begin{array}{l}\text { Chat- } \\
\text { raum }\end{array}$ & $\begin{array}{l}\text { Linien } \\
\text { ink1. } \\
\text { Status- } \\
\text { und } \\
\text { Ereignis- } \\
\text { zeilen }\end{array}$ & Beiträge & $\begin{array}{l}\text { Beiträge } \\
\text { ohne } \\
\text { Doppel } \\
\text { chan- } \\
\text { nels" }\end{array}$ & $\begin{array}{l}\text { "left } \\
\text { / Auftritt }\end{array}$ & $\begin{array}{l}\text { Beiträge } \\
\text { Namen }\end{array}$ & $\begin{array}{l}\text { Wörter } \\
\text { (inkl. } \\
\text { Status- } \\
\text { zeilen) }\end{array}$ & $\begin{array}{l}\text { Wörter } \\
\text { (nur } \\
\text { Text) }\end{array}$ & $\begin{array}{l}\varnothing \text { Bei- } \\
\text { trags- } \\
\text { länge }\end{array}$ \\
\hline \#zuerich & 62726 & 32372 & 29220 & 6216 & 5.21 & 3789 & 369358 & 122846 & 3.79 \\
\hline \#bern & 74441 & 41980 & 37485 & 6242 & 6.73 & 3680 & 425569 & 154900 & 3.69 \\
\hline \#wallis & 44553 & 27265 & 24871 & 1796 & 15.18 & 1143 & 244401 & 96239 & 3.53 \\
\hline $\begin{array}{l}\text { \#grau- } \\
\text { buenden }\end{array}$ & 37111 & 20824 & 19129 & 2998 & 6.95 & 1666 & 214743 & 81233 & 3.90 \\
\hline Total & 218831 & 122441 & 110705 & 17252 & 7.10 & 10278 & 1254071 & 455218 & $3.72^{4}$ \\
\hline
\end{tabular}

Tabelle 2: Grunddaten zur Datenbasis

Für die Analyse wurden jeweils identische Linien in der Protokolldatei ausgeblendet. Damit wurden Mehrfacheinträge derselben ChatterInnen gelöscht. Das betrifft einerseits Kurzkommentare, die oft nicht Wortstatus haben, andererseits längere Werbebeiträge, die oft mit Skripts erstellt wurden. Nicht ausgeschlossen werden aber gleiche Einträge verschiedener ChatterInnen, sofern sie unter unterschiedlichen Namen auftreten. Im Gesamtkorpus werden so rund 11736 , d. h. rund $10 \%$ aller Beiträge ausgeschaltet, die etwa so aussehen:

\footnotetext{
${ }^{4}$ Mit 3.72 Wörtern pro Turn sind diese Beiträge nochmals um einiges kürzer als dies von Runkehl et al. (1998: 85) mit durchschnittlich 4.8 Wörter pro Turn belegt wird.
} 


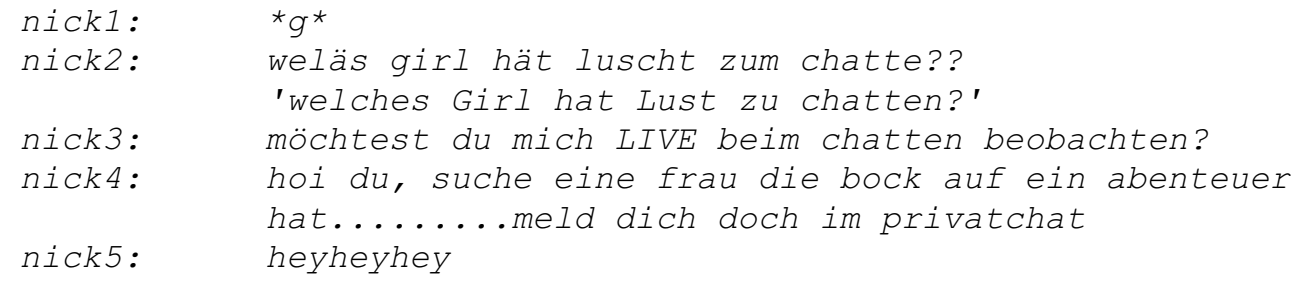

(Alle Beispiele aus \#zuerich)

Vereinzelt kommen in allen Chaträumen auch andere Sprachen vor. Im Chatraum \#wallis vor allem Französisch, im Chatraum \#graubuenden Romanisch, überall immer wieder Englisch mit interessanten Code Switches -, häufig auch Italienisch, vereinzelt Portugiesisch, slawische Sprachen oder Türkisch. Diese Belege wurden nicht einzeln aus den Daten ausgefiltert. Sie machen jedoch nur einen geringen Anteil der Belege aus.

\section{$3 \quad$ Mundartverwendung}

Schon mehrfach wurde darauf hingewiesen, dass in Schweizer Chaträumen mehr Mundart geschrieben wird als in deutschen oder österreichischen Chats. Die folgende Tabelle gibt einige Hinweise auf die Verwendung verschiedener Varietäten. Gegenübergestellt werden jeweils standardsprachliche und mundartliche Schreibungen bzw. Varianten in mundartlicher oder standardsprachlicher Verwendung. Diese Verwendung wird ermittelt über die Schreibung des entsprechenden Wortes oder, falls dies bei gleicher Schreibung nicht möglich ist, durch die Schreibung der Umgebung innerhalb desselben Turns.

\begin{tabular}{|c|c|c|c|}
\hline Belegwort & $\begin{array}{l}\text { Anzahl Belege } \\
\text { gesamt }\end{array}$ & mundartlich & Mundartanteil \\
\hline "nich(t)" - "nöd/nid/ned/nid/nit"5 & 6168 & 5318 & $86.1 \%$ \\
\hline $\begin{array}{l}\text { "chatten" mit allen Verbalformen in standard- } \\
\text { sprachlicher - mundartlicher Verwendung }\end{array}$ & 1699 & 1523 & $89.6 \%$ \\
\hline $\begin{array}{l}\text { "machen" in standardsprachlicher - } \\
\text { mundartlicher Verwendung }\end{array}$ & 1230 & 1037 & $84.3 \%$ \\
\hline $\begin{array}{l}\text { "schreiben" in standardsprachlicher - } \\
\text { mundartlicher Verwendung }\end{array}$ & 185 & 148 & $80.2 \%$ \\
\hline $\begin{array}{l}\text { Plural von "sein" in standardsprachlicher - } \\
\text { mundartlicher Verwendung }\end{array}$ & 1617 & 1382 & $85.5 \%$ \\
\hline $\begin{array}{l}\text { 2./3. Sg. von "sein" in standardsprachlicher - } \\
\text { mundartlicher Verwendung }\end{array}$ & 11065 & 9846 & $89.0 \%$ \\
\hline $\begin{array}{l}\text { "mich" in standardsprachlicher - } \\
\text { mundartlicher Verwendung }\end{array}$ & 1484 & 1253 & $84.4 \%$ \\
\hline
\end{tabular}

Tabelle 3: Mundartliche Verwendung verschiedener Wörter und Wortformen in allen vier Chaträumen

\footnotetext{
${ }^{5}$ Die Vertretung von «nicht» eignet sich gut als Indikator für Regionalität, da sie relativ häufig vorkommt, eine beschränkte Variabilität hat und im gesamten deutschen Sprachraum umgangssprachlich regionale Varianten aufweist. Arbeiten zur Regionaliät im Chat (Kelle 2002; Christen u. a. i. Dr.) nehmen diese Variable auf. Sie ist deshalb auch hier gegeben, um überregionale Vergleiche zwischen verschiedenen Datensammlungen zu ermöglichen.
} 

und Verschriftung in schweizerdeutschen Chats

\begin{tabular}{|l|l|l|l|l|l|l|l|l|}
\hline Chatroom & "nicht" & "chatten" & "schreiben" & "machen" & Pl. "sein" & Sg. "sein" & "mich" & gesamt \\
\hline ZH & $76.13 \%$ & $85.31 \%$ & $63.01 \%$ & $76.92 \%$ & $80.24 \%$ & $80.88 \%$ & $78.72 \%$ & $79.36 \%$ \\
\hline BE & $90.10 \%$ & $89.12 \%$ & $87.14 \%$ & $89.16 \%$ & $91.90 \%$ & $91.58 \%$ & $84.52 \%$ & $90.29 \%$ \\
\hline WS & $88.82 \%$ & $89.02 \%$ & $69.57 \%$ & $80.65 \%$ & $77.54 \%$ & $92.51 \%$ & $88.55 \%$ & $89.31 \%$ \\
\hline GR & $89.12 \%$ & $96.03 \%$ & $73.68 \%$ & $95.71 \%$ & $94.87 \%$ & $90.77 \%$ & $89.20 \%$ & $91.16 \%$ \\
\hline Total & $86.19 \%$ & $89.62 \%$ & $74.05 \%$ & $84.31 \%$ & $85.47 \%$ & $88.98 \%$ & $84.43 \%$ & $87.40 \%$ \\
\hline
\end{tabular}

Tabelle 4: Mundartanteil der Markiervariablen in den einzelnen Chaträumen

Die Tabellen 3 und 4 zeigen, dass der mundartliche Anteil im Chat bei allen ausgewählten Wörtern, die als Indizes verstanden werden können, normalerweise deutlich über $80 \%$ liegt. In den Chaträumen \#bern, \#wallis und \#graubuenden liegt der Gesamtprozentsatz bei $90 \%$, nur im \#zuerich-Chatroom liegt er mit $79 \%$ deutlich darunter. Dieser Anteil ist einiges höher als der Anteil von etwa $30 \%$, den Christen, Tophinke, Ziegler (i. Dr.) angeben, was vermutlich damit zu tun hat, dass der \#swissonline-Chatroom, den Christen u. a. untersuchen, in der Schweiz eine gesamtschweizerische Verbreitung hat, während die hier untersuchten Räume ein kleineres Gebiet abdecken. Der Unterschied wäre somit so zu interpretieren, dass sogar innerhalb der Schweiz die Standardsprache eine überregionale(re) Kommunikationsfunktion übernimmt, während die regionale Mundart, die sich in den hier untersuchten Chaträumen zeigt, viel stärker die regionale Bindung vermittelt. Dass der Mundartanteil im Zürcher Chat etwas tiefer liegt als in den anderen Kanälen, kann gerade auch damit zu tun haben. Es kann auch sein, dass der hohe Mundartanteil mit dem Alter der ChatterInnen zu tun hat. Die ChatterInnen in den hier untersuchten Chaträumen geben zu einem Großteil an, der Altersklasse zwischen 15 und 22 anzugehören, sei es, dass sie einen sprechenden Namen tragen (chatboy_22, flirtgirl_18) oder dass sie auf die Frage, wie alt sie seien, ein Alter dieser Klasse angeben. Ältere sind für einen Chat dann oft zu alt. Die Frage

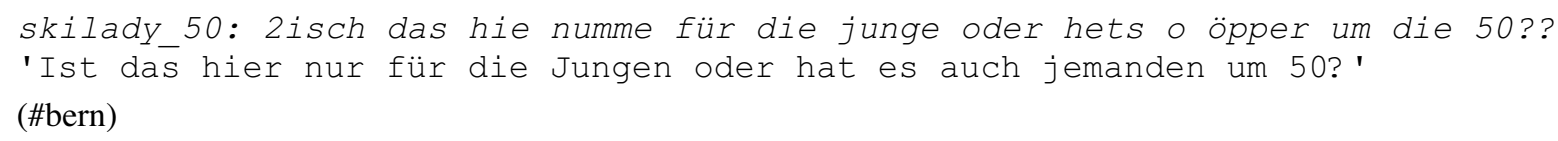

bleibt auf jeden Fall unbeantwortet und skilady_50 verlässt den Chatroom bald wieder. Zudem werden häufig schulische Hintergründe diskutiert oder zumindest angesprochen. Teilweise werden sogar 'ältere Semester' explizit ausgeladen:

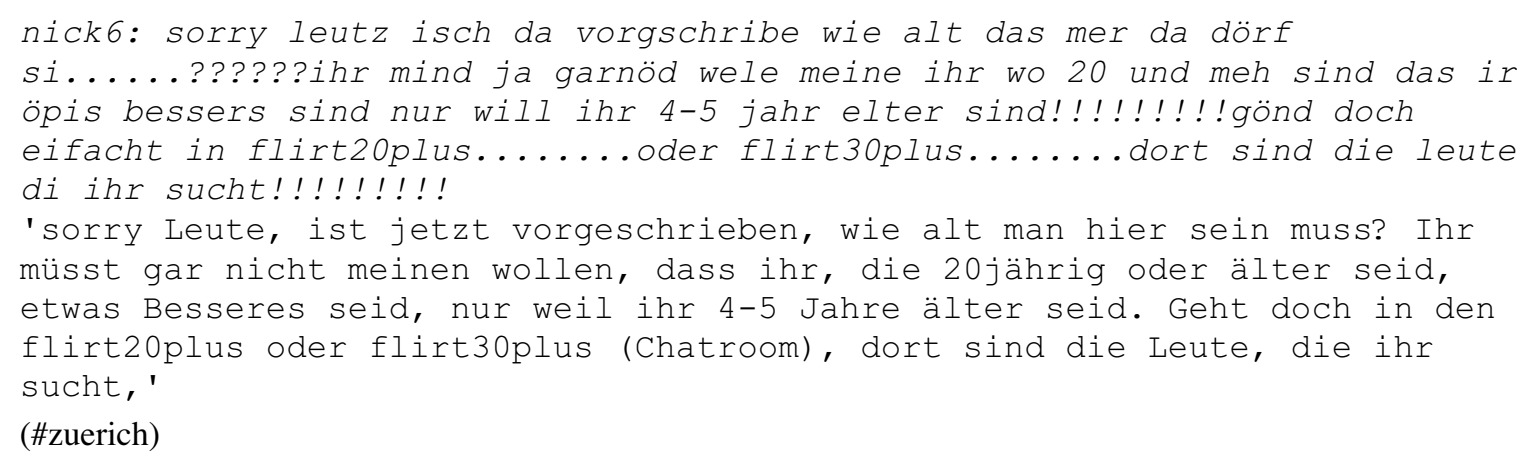

Übrigens ein schönes Beispiel für Code-switching: der Chatter wechselt in der letzten Aussage, wo er die älteren Semester zum Verlassen des Chatraums auffordert, zur Standardsprache - eine implizite Reklamierung der Mundart für die eigene Altersgruppe, während den älteren Semestern die Standardsprache zugeordnet wird. 
Diese Altersangaben müssen natürlich nicht dem tatsächlichen Alter entsprechen. Im unsystematischen Vergleich mit anderen Kanälen, die ein älteres Publikum ansprechen, erscheint in den untersuchten Räumen die Mundart aber deutlich stärker vertreten.

\section{$4 \quad$ Schreibung zum Ersten: Schreibtraditionen}

Es wurde oben darauf hingewiesen, dass sich das mundartliche Schreiben im Chat wenig um Normen kümmert. Schon das Faktum, dass nicht die Standardsprache geschrieben wird, deutet darauf hin, dass ein informeller Rahmen vorliegt, in dem Normen weniger einzuhalten sind. Somit stellt die Mundartschreibung in der Schweiz eine gute Möglichkeit dar, aus dieser Norm der Standardsprache als Sprache der Schule auszubrechen. Es ist also nicht zu erwarten, dass an die Stelle der schriftsprachlichen Norm eine Normierung der Dialektschreibung tritt. Aschwanden (2002: 61) vermutet, dass die meist jugendlichen ChatterInnen die bestehenden Normierungsversuche zur Mundartschreibung nicht kennen. Gleichzeitig stehen sie auch nicht in einer mundartlichen Schreibtradition der Dialektliteratur, die heute vor allem ältere Leser anspricht und von jüngeren kaum rezipiert wird (Schmid 2003). Das lässt sich beispielsweise anhand der Verwendung des Graphems $\langle\mathrm{y}\rangle{ }^{6}$ für das geschlossene [i/ii] zeigen. Die Berner Schreibtradition, die sich auf die vor-neuhochdeutsche Schweizer Schriftsprache stützt und die von Marti (1972) kodifiziert wurde, zeigt weitgehend diese y-Schreibung. Für die ostschweizerdeutsche Mundartliteratur wird sie viel seltener verwendet. Sie ist aber von Dieth (1938/1986, 31) ebenfalls zugelassen. Die geloggten Chats zeigen die y-Schreibung für [i/ii] kaum, wie aus den folgenden Tabellen ersichtlich ist:

\begin{tabular}{|l|l|l|l|}
\hline Belegwort & Anzahl Belege gesamt & davon mit $\langle\mathrm{y}\rangle$ & Anteil \\
\hline schribe/gschribe 'schreiben' & 107 & 0 & $0.0 \%$ \\
\hline chly/chli/chlei 'klein/wenig' & 906 & 11 & $1.2 \%$ \\
\hline mys/sys 'mein/sein' & 445 & 15 & $3.4 \%$ \\
\hline sy/si - gsy/gsi 'sein - gewesen' & 1747 & 31 & $1.7 \%$ \\
\hline
\end{tabular}

Tabelle 5: $y$-Schreibung für [i/ii] in allen vier Chaträumen

Die y-Schreibung, wie sie in der Mundartliteratur, vor allem im Berndeutschen vorkommt, findet sich also in Chats nur in einem sehr geringen Maß (Tabelle 5). Die Analyse nach den einzelnen Chaträumen zeigt zudem, dass diese nur im Berner Chatraum vorkommen, während sie sonst gänzlich fehlen.

\begin{tabular}{|l|l|l|l|}
\hline Belegwort & Anzahl Belege gesamt & davon mit $\langle\mathrm{y}\rangle$ & Anteil \\
\hline schribe/gschribe 'schreiben' & 48 & 0 & $0.0 \%$ \\
\hline chly/chli/chlei 'klein/wenig' & 530 & 11 & $2.0 \%$ \\
\hline mys/sys 'mein/sein' & 219 & 15 & $6.8 \%$ \\
\hline sy/si - gsy/gsi 'sein - gewesen' & 846 & 31 & $3.6 \%$ \\
\hline
\end{tabular}

Tabelle 6: y-Schreibung für [i/ii] im Chatraum \#bern

\footnotetext{
${ }^{6}$ Die Schreibung 〈yy〉 für [i:] hat sich weniger durchgesetzt. Sie zeigt sich vor allem in baseldeutschen Texten, während 〈y〉 sich im Berndeutschen "für die Doppelschreibung (Länge) nicht eignet" (Marti 1972: 33), weil das Schriftbild ungewohnt sei.
} 

und Verschriftung in schweizerdeutschen Chats

Die Schreibtradition ist in diesen Daten also doch noch festzustellen, aber auch im Berner Chat (Tabelle 6) sind diese Schreibungen eher als marginal zu bezeichnen.

\section{$5 \quad$ Regionalität}

Im Folgenden soll die regionale Verankerung dieser Chats auch in sprachlicher Hinsicht anhand zweier Beispiele untersucht werden. Es ist klar, dass sich in diesen Chaträumen, die als regional definiert sind, nicht nur Teilnehmer aus der Region einfinden, sondern dass auch Fremde vorgeben können, aus der entsprechenden Region zu stammen. Teilweise kommen auch Fremde als 'Besucher', die sich dann explizit als Fremde zu erkennen geben, sei es mit dem Namen, wie *buendner* im \#wallis-Chatroom, oder im Text selbst, wo sich die Identifikation über die geografische Selbstzuordnung und über die sprachliche Selbstinszenierung manifestieren kann.

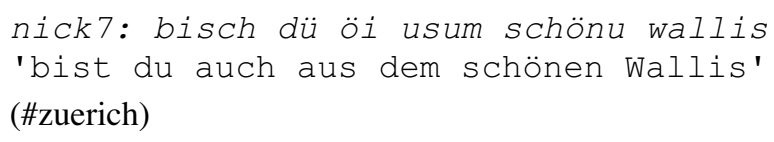

Zudem können Fremde vorgeben, einheimisch zu sein, und das sogar auch sprachlich tun, wie Aschwanden (2001: 61) und Kelle (2002) belegen. Trotz dieser Bedenken lässt sich eine klare regionale Verortung der untersuchten Chaträume vornehmen, wie im Folgenden anhand einiger ausgewählter morphologischer Kriterien gezeigt werden soll. Die standardsprachlichen Varianten werden nicht mehr in die Analyse einbezogen.

\section{Endungsvokal im Infinitiv}

Das Schweizerdeutsche zeigt eine deutliche Gliederung im Bezug auf den Endungsvokalismus der Normalverben, das sind diejenigen ehemals starken und schwachen Verben, die einen mehrsilbigen Infinitiv haben (im Gegensatz zu den Kurzverben sy, haa, gaa, staa, laa, tue, faa ...). Die Karte zur Infinitivendung im Sprachatlas der deutschen Schweiz (SDS III 1) vermittelt deutlich eine als Reliktstaffelung bezeichnete Verteilung. Die alten Vollvokale sind im Wallis erhalten - Teile des Walliserdeutschen unterscheiden sogar noch die alten schwachen Verben -, in den übrigen südlichen Mundarten sind die Endungen in einen Reduktionsvokal zusammengefallen, der in zentralen und westlichen Bereichen eine [æ]-Färbung hat, während in Graubünden eine [a]-Qualität erscheint. Die nördlichen Teile der Schweiz zeigen einen stärker zentralisierten Schwa-Laut. 


\begin{tabular}{|l|l|l|l|l|l|}
\hline & mache & mach $\ddot{a}$ & machu & macha & Total \\
\hline ZH & 34 & 8 & 0 & 0 & 42 \\
\hline BE & 55 & 28 & 0 & 0 & 83 \\
\hline WS & 11 & 0 & 28 & 0 & 39 \\
\hline GR & 9 & 0 & 0 & 13 & 22 \\
\hline Total & 109 & 36 & 28 & 13 & 186 \\
\hline & & & & & \\
\hline ZH & $81.0 \%$ & $19.0 \%$ & $0.0 \%$ & $0.0 \%$ & $100.0 \%$ \\
\hline BE & $66.3 \%$ & $33.7 \%$ & $0.0 \%$ & $0.0 \%$ & $100.0 \%$ \\
\hline WS & $28.2 \%$ & $0.0 \%$ & $71.8 \%$ & $0.0 \%$ & $100.0 \%$ \\
\hline GR & $40.9 \%$ & $0.0 \%$ & $0.0 \%$ & $59.1 \%$ & $100.0 \%$ \\
\hline Total & $58.6 \%$ & $19.4 \%$ & $15.1 \%$ & $7.0 \%$ & $100.0 \%$ \\
\hline
\end{tabular}

Tabelle 7: "machen": Infinitiv Endungsvokal - Anzahl und Anteil der Belege in den vier Chaträumen

Wenn nun die Belege im Chat analysiert werden, so spiegelt sich diese traditionelle Struktur noch deutlich. Aus Tabelle 7 ist ersichtlich, dass die alpinen Formen mit $\langle-\mathrm{u}\rangle$, bzw. $\langle-\mathrm{a}\rangle$ nur je in dem Gebiet vorkommen, in dem schon der SDS entsprechende Lautungen belegt. Sie machen da jeweils den größten Teil aller Formen aus. Daneben erscheint jeweils auch die Form mit 〈e〉 als unmarkiertes Graphem für den Reduktionsvokal. In den Chaträumen \#bern und \#zuerich findet sich neben dem hauptsächlich verwendeten 〈-e〉 auch eine Graphie mit 〈-̈̈〉. Dafür bieten sich zwei Erklärungen an: Auf dialektologischem Hintergrund lässt sich einerseits postulieren, dass die volleren Werte, die teilweise in Zürich und Bern neben dem Schwa-Laut vorkommen, durch dieses <-ä〉 markiert werden, oder andererseits, dass hier ChatterInnen aus der Zentralschweiz, die solche Werte haben, einen größeren Anteil der TeilnehmerInnen ausmachen. Neben dieser dialektologischen Erklärung ist auch eine graphematische Erklärung möglich und zudem wahrscheinlicher. Das Endungsschwa in den mittelländischen Mundarten ist im Allgemeinen peripherer realisiert als in der deutschen Standardlautung. Diesem Unterschied der beiden Lautungen tragen die SchreiberInnen Rechnung, indem sie die Abweichung von der Standardsprache mit <-ä〉 markieren, was eine häufige Lösung auch in anderen Kontexten darstellt (cf. Lötscher 1989). Diese Hypothese wird gestützt dadurch, dass diese Schreibungen in den Chaträumen \#wallis und \#graubuenden nicht vorkommen, wo andere Vollformen Verwendung finden. Gestützt wird die äSchreibung durch eine Funktion als Dialektmarker, wie sie von Christen (eingereicht) beschrieben wird. An Stellen, wo der Abstand zur Standardsprache relativ klein ist und die Dialektalität der Turns nicht eindeutig vermittelt werden kann, kann ein 〈ä〉 die Dialektalität markieren. Christen vermutet, dass diese Funktion vor allem in kürzeren Beiträgen zum Tragen kommt, wo keine anderen Dialektmarker erscheinen. Das ist in den untersuchten Daten nicht der Fall, denn der Infinitiv erscheint jeweils am Schluss eines Satzes; in allen Fällen finden sich vorher schon mehrere graphematische, morphologische oder lexikalische Dialektmerkmale. So kann vermutet werden, dass eine phonetische Distanz zur Standardsprache markiert wird, nicht jedoch unbedingt der phonetische Lautwert des Vollvokals beschrieben wird.

Die beschriebene Verteilung findet sich nicht nur bei "machen", einem Normalverb, das im Althochdeutschen noch der 2. Klasse der schwachen Verben auf -ôn angehört hatte, sondern auch bei Neologismen. Der Infinitiv von "chatten" kommt sehr häufig vor. Dabei handelt es 

und Verschriftung in schweizerdeutschen Chats

sich um ein junges Fremdwort aus dem Englischen, das selbstverständlich in die Mundart aufgenommen und morphologisch dem mundartlichen System angepasst wird (Tabelle 8).

\begin{tabular}{|l|l|l|l|l|l|l|l|l|}
\hline Endung auf & $-e$ & $-\ddot{a}$ & $-u$ & $-a$ & $-o$ & $-\ddot{o}$ & $-e n$ & Total \\
\hline ZH & 271 & 86 & 0 & 7 & 1 & 0 & 14 & 379 \\
\hline BE & 298 & 125 & 0 & 6 & 0 & 1 & 12 & 442 \\
\hline WS & 104 & 1 & 139 & 2 & 19 & 0 & 13 & 278 \\
\hline GR & 122 & 7 & 0 & 216 & 0 & 0 & 6 & 351 \\
\hline Total & 795 & 219 & 139 & 231 & 20 & 1 & 45 & 1450 \\
\hline & & & & & & & & \\
\hline ZH & $71.5 \%$ & $22.7 \%$ & $0.0 \%$ & $1.8 \%$ & $0.3 \%$ & $0.0 \%$ & $3.7 \%$ & $100.0 \%$ \\
\hline BE & $67.4 \%$ & $28.3 \%$ & $0.0 \%$ & $1.4 \%$ & $0.0 \%$ & $0.2 \%$ & $2.7 \%$ & $100.0 \%$ \\
\hline WS & $37.4 \%$ & $0.4 \%$ & $50.0 \%$ & $0.7 \%$ & $6.8 \%$ & $0.0 \%$ & $4.7 \%$ & $100.0 \%$ \\
\hline GR & $34.8 \%$ & $2.0 \%$ & $0.0 \%$ & $61.5 \%$ & $0.0 \%$ & $0.0 \%$ & $1.7 \%$ & $100.0 \%$ \\
\hline Total & $54.7 \%$ & $15.1 \%$ & $9.6 \%$ & $16.2 \%$ & $1.4 \%$ & $0.1 \%$ & $3.1 \%$ & $100.0 \%$ \\
\hline
\end{tabular}

Tabelle 8: "chatten": Infinitiv Endungsvokal - Anzahl und Anteil der Belege in den vier Chaträumen

Neben den schon bekannten Endungen erscheinen noch solche auf <-en〉 - vermutlich eine Konfliktform aus der Standardsprache -, zudem ein einzelner Beleg für <-ö〉 - vermutlich ein Verschreiber für $<-\ddot{a}\rangle$, neben dem es auf der Tastatur erscheint. Zudem erscheint die Endung auf $<-0\rangle$ mit einer Ausnahme im Chatraum \#zuerich nur im Chatraum \#wallis mit immerhin 19 Belegen, das lässt auf eine gewisse Systematik schließen. Der Blick in die SDS-Karte (SDS III 1) bestätigt die Altertümlichkeit dieser Form. Neben der Vollform auf -u können nämlich auch seltener Formen auf -o erscheinen. Die im Chat vereinzelt vorgefundene Form ist also durch die alten Daten gestützt. Für den Rest ergibt sich ein ähnliches Bild wie bei "machen": 〈-e ist die häufigste Endung in den Chaträumen \#zuerich und \#bern, wo daneben auch <-ä〉 vorkommt. Die e-Schreibung ist jeweils die zweithäufigste Form in den Chaträumen \#wallis und \#graubuenden, in denen <-ä〉 kaum vorkommt, während die alpinen Formen auf 〈-u〉 und $<-a 〉$ in den mittelländischen Chatrooms kaum vertreten sind.

\section{Endungskonsonant bei "sein" 2./3.Sg.}

Für die 2. und 3. Person Singular ist im SDS in der Nordostschweiz und den südlichen Alpenmundarten weitgehend die Flexion auf $-t$ vor Folgevokal ausgewiesen (SDS III 42). In der Untersuchung dialektaler Alltagssprache macht Christen (1998: 159) darauf aufmerksam, dass schon die teilweise archaisierenden Mundartgrammatiken dieses - $t$ nicht mehr erwähnen. Auch in ihrer empirischen Untersuchung ist das Flexions-t nur noch einmal bei einem Walliser Sprecher vertreten. Diesem t-Verlust bei der 2./3. Singular von "sein" schließt sich die t-Reduktion bei der 2. Person Singular der Normalverben an. Christen schließt daraus aufgrund der unten dargestellten Daten etwas vorschnell -, dass "die t-Formen bereits in der heutigen Deutschschweiz nicht mehr bekannt sind und alltagsweltlich ausschließlich als veraltete Formen betrachtet werden" (Christen 1998: 161).

Vor diesem Hintergrund ist es überraschend, dass die t-Formen in den Chats erscheinen, wo vor allem jüngere Personen auftreten. Tabelle 9 dokumentiert das Vorkommen dieser Formen, die in Zürich nie, in Bern ganz selten, jedoch häufiger in den Chaträumen \#wallis und 
\#graubuenden belegt sind. Dabei erstaunt die Verteilung: Während nämlich im Chatraum \#wallis fast ausschließlich die 2. Person mit Dentalsuffix verwendet wird, ist es im Chatraum \#graubuenden ebenso fast ausschließlich die 3. Person. Diese Verteilung lässt sich aus den Daten des SDS nicht erklären, sind doch für unterschiedliches Verhalten der 2. und 3. Person Belege aus beiden Gebieten erwähnt.

\begin{tabular}{|l|l|l|l|l|l|l|l|}
\hline & bisch & bischt & isch & ischt & Total & Endungs-t & \\
\hline ZH & 800 & 0 & 1413 & 0 & 2213 & 0 & $0.0 \%$ \\
\hline BE & 1158 & 6 & 2155 & 0 & 3319 & 6 & $0.2 \%$ \\
\hline WS & 684 & 75 & 1537 & 1 & 2297 & 76 & $3.3 \%$ \\
\hline GR & 694 & 2 & 1186 & 135 & 2017 & 137 & $6.8 \%$ \\
\hline Total & 3336 & 83 & 6291 & 136 & 9846 & 219 & $2.2 \%$ \\
\hline
\end{tabular}

Tabelle 9: 2. und 3. Sg. von "sein" in den vier Chaträumen

Während also das Endungs-t in "sein" noch vorkommt, ist es bei der 2. Singular der Normalverben völlig verschwunden: Von den 187 Belegen für "du machst" zeigt ein einziger aus dem Chatraum \#wallis eine Plosivendung, bei "du chattest" ist es nie vertreten. Die Belege lassen aber insgesamt erkennen, dass zumindest in den Alpinmundarten die t-Flexion noch nicht völlig verschwunden ist und sogar in modernen Kommunikationsformen unter jungen Leuten noch Verwendung findet, wenn auch auf sehr tiefem Niveau. Dass dabei in beiden Regionen, in denen diese Form noch vorkommt, eine neue Personendifferenzierung gemacht wird, ist ein spannendes Phänomen für die Neuorganisation von Variation und in der deutlichen Verteilung durchaus überraschend. Da diese neue Morphologisierung des - $t$ im Wallis auf die 2. Person von "sein" beschränkt bleibt, ist zu vermuten, dass sie sich nicht durchsetzen kann. Die Verwendung des - $t$ in der 3. Person von "sein" in Graubünden ist dagegen durch die t-Endung der übrigen Verben gestützt und hat dadurch eine etwas größere Chance, sich zu etablieren.

\section{Schreibung zum Zweiten: Neologismen}

Es wurde zum einen gezeigt, dass die Schreibtradition kaum Eingang in die Schreibung der ChatterInnen findet, und zum andern, dass sich Variation zu einem großen Anteil auf lautliche Unterschiede zurückführen lässt. Gleichzeitig ist aber auch in den einzelnen Chaträumen eine große Variation zu finden. Die Schreibung der Mundart im Chat bleibt also eine mehr oder weniger individuelle Verschriftung einer gesprochenen Varietät. Dies kann auch in der Schreibung von Neologismen gezeigt werden. Es wurde oben schon darauf hingewiesen, dass "chatten" in die mundartliche Morphologie eingegliedert wird. Die mit Erbwörtern vergleichbare Verteilung des Endungsvokalismus ist ein deutliches Indiz für diese Eingliederung. Hier soll aber zudem noch der Stamm dieses Neologismus untersucht werden. Die Schreibung von Neologismen ist besonders interessant, weil sie sich stark an der (mundartlichen) Lautung orientiert, denn die Schreibung ist auch in der deutschen Standardsprache noch nicht etabliert. Diese orientiert sich zwar weit gehend an der englischen Schreibung, der Anlaut mit 〈ch-〉 und die a-Schreibung für [æ] wird beibehalten, aber bei der Anzahl der Konsonanten ist sich die deutsche Sprachgemeinschaft nicht ganz einig. Neben 

und Verschriftung in schweizerdeutschen Chats

614'000 deutschen Web-Seiten mit «chatten〉 gibt es auch 103'000 mit «chaten>. ${ }^{7}$ Bei der Mundartschreibung zeigt sich zusätzliche Varianz bei der Schreibung des Anlauts und der Vokalqualität des Stammvokals.

\begin{tabular}{|l|l|l|l|l|}
\hline & $t s c h$ & $s c h$ & $c h$ & Total \\
\hline ZH & 5 & 0 & 384 & 389 \\
\hline BE & 2 & 0 & 471 & 473 \\
\hline WS & 10 & 1 & 275 & 286 \\
\hline GR & 1 & 0 & 362 & 363 \\
\hline Total & 18 & 1 & 1492 & 1511 \\
\hline
\end{tabular}

Tabelle 10: "chatten": Anlaut aller mundartlichen Belege ohne Partizipien

Der englische Anlaut [t $\mathrm{t}]$, mit ch-Schreibung, wird auch in der Mundartschreibung weitgehend beibehalten, jedoch finden sich auch vereinzelt tsch-Schreibungen, die lauttreuer sind. Im \#wallis-Chat machen diese Schreibungen rund $3.5 \%$ aller Belege aus.

\begin{tabular}{|l|l|l|l|l|}
\hline & $a$ & $\ddot{a}$ & $e$ & \\
\hline ZH & 324 & 63 & 2 & 389 \\
\hline BE & 356 & 116 & 0 & 472 \\
\hline WS & 260 & 15 & 11 & 286 \\
\hline GR & 325 & 32 & 6 & 363 \\
\hline Total & 1265 & 226 & 19 & 1510 \\
\hline & & & & \\
\hline ZH & $83.29 \%$ & $16.20 \%$ & $0.51 \%$ & $100.00 \%$ \\
\hline BE & $75.42 \%$ & $24.58 \%$ & $0.00 \%$ & $100.00 \%$ \\
\hline WS & $90.91 \%$ & $5.24 \%$ & $3.85 \%$ & $100.00 \%$ \\
\hline GR & $89.53 \%$ & $8.82 \%$ & $1.65 \%$ & $100.00 \%$ \\
\hline Total & $83.77 \%$ & $14.97 \%$ & $1.26 \%$ & $100.00 \%$ \\
\hline
\end{tabular}

Tabelle 11: "chatten": Stammvokal aller mundartlichen Belege

Auch bei der Stammvokalschreibung wird mehrheitlich an der englischen Schreibung festgehalten (Tabelle 11), obwohl der normale Lautwert des <a〉 weder in der Mundart noch in der Standardsprache realisiert wird. In gut $16 \%$ aller Belege wird aber von dieser Schreibung abgewichen und die Abweichung der Standardschreibung von der Lautung markiert. Mehrheitlich wird dafür das Graphem 〈ä〉 verwendet, zu einem geringen Prozentsatz auch 〈e〉. Mit der e-/ä-Schreibung ist eines der häufig genannten Verschriftungsprobleme angesprochen.

Die mittelländischen Chats markieren diese lautliche Abweichung häufiger als die alpinmundartlichen Chats. Die ä-Graphie ist im Berner und Zürcher Chat generell häufiger zu finden, wie oben gezeigt auch bei der Schreibung des Reduktionsvokals in der Infinitivendung. Der große Anteil der ä-Schreibungen im Berner Chat lässt sich mit der mundartlichen Realisierung erklären, die in Bern meist mit überoffenem [æ] erfolgt, das auch in der Schreibung von Erbwörtern mit 〈ä〉 wiedergegeben wird. Eine Konkurrenz zur Schreibung 〈e〉 ist kaum gegeben. In Zürich sieht die Situation insofern anders aus, als da ein dreistufiges System der /æ, $\varepsilon$, e/ Laute besteht und die Zuordnung von / $\varepsilon /$ zum Graphem <ä〉 oder 〈e〉 immer zu Konflikten mit den Phonemen /æ/ oder /e/ führt. Für Mundartschriftsteller

\footnotetext{
${ }^{7}$ Google-Suche vom 4.11.2002
} 
hat Lerch (1971) gezeigt, dass bei der Verwendung eines zweistufigen Graphemsystems für ein dreistufiges Lautsystem mehrheitlich die Graphie 〈e〉 für den mittleren Laut verwendet wird. Da in Zürich "chatten" sowohl mit [æ] als auch mit [ع] zu hören ist, ist erstaunlich, dass nicht mehr e-Graphien auftreten. Zu vermuten ist, dass für eine e-Schreibung die graphische Distanz zur gewohnten a-Graphie zu groß ist und gleichzeitig auch die Distanz zur [e]Lautung, somit also der ä-Graphie der Vorzug gegeben wird. Im Chatraum \#wallis ist der eAnteil etwas größer, aber auch hier fast nicht bemerkbar.

\begin{tabular}{|l|l|l|l|}
\hline & $t$ & $t t$ & Total \\
\hline ZH & 130 & 259 & 389 \\
\hline BE & 100 & 373 & 473 \\
\hline WS & 31 & 255 & 286 \\
\hline GR & 86 & 277 & 363 \\
\hline Total & 347 & 1164 & 1511 \\
\hline & & & \\
\hline ZH & $33.42 \%$ & $66.58 \%$ & $100.00 \%$ \\
\hline BE & $21.14 \%$ & $78.86 \%$ & $100.00 \%$ \\
\hline WS & $10.84 \%$ & $89.16 \%$ & $100.00 \%$ \\
\hline GR & $23.69 \%$ & $76.31 \%$ & $100.00 \%$ \\
\hline Total & $22.96 \%$ & $77.04 \%$ & $100.00 \%$ \\
\hline
\end{tabular}

Tabelle 12: "chatten": Konsonantenschreibung aller mundartlichen Belege

Aus Tabelle 12 ist die Verteilung der Doppelschreibung von 〈 $t\rangle$ ersichtlich. Wie auf den standardsprachlichen Webseiten ist im Chat eine erhebliche Variation festzustellen. Diese ist kaum auf mundartliche Variation im Konsonantismus zurückzuführen, denn eine FortisGeminaten-Opposition existiert nicht. Die Doppelschreibung von Plosiven folgt damit im Allgemeinen der Standardschreibung und damit dem Prinzip, dass Doppelkonsonanten die Kürze des vorangehenden Vokals anzeigen. Die mehrheitliche Verwendung des 〈 $t \mathrm{t}\rangle$ entspricht diesem Prinzip. Die Abweichungen davon können wie in der Standardsprache mit Analogie zum Substantiv Chat erklärt werden oder mit dem Schreibprinzip, dass ein Laut mit einem Buchstaben wiedergegeben werden soll. Die Variation zwischen den Chaträumen ist damit aber nicht geklärt.

\section{$9 \quad$ Fazit}

Die Zusammenstellung hat gezeigt, dass die Mundartverwendung im Netz für Seiten aus der deutschsprachigen Schweiz mit einem Anteil von $20 \%$ nicht unüblich ist. In den untersuchten regionalen Chaträumen steigt dieser Anteil auf rund $90 \%$, außer im Zürcher Chatraum mit $80 \%$ Mundartanteil. Die Mundartverwendung im Chat wird erklärt mit einer Tendenz, für den konzeptionellen Nähebereich auch in schriftlicher Kommunikation die Mundart zu verwenden. Zudem spricht die durchgehende Dialektkompetenz in der Deutschschweiz gekoppelt mit der Freiheit, ohne schulisch gedrillte Regeln schreiben zu dürfen, für eine vermehrte Verwendung der Mundart. Die umfangreichen Daten aus den öffentlichen Chaträumen zeigen auch, dass die mundartlichen Schreibtraditionen, die wesentlich über die häufig als altertümlich empfundene - Mundartliteratur vermittelt werden, nur wenig Einfluss auf die spontane Mundartschreibung der jugendlichen ChatterInnen haben. Die 
Mundartschreibung nimmt also kaum den Weg einer Vereinheitlichung, sondern bleibt die mehr oder weniger individuelle Verschriftung einer gesprochenen Varietät.

Deswegen lassen sich die klassischen Dialektunterschiede auch im Chat finden. Die untersuchten Daten zeigen die im SDS belegte Verteilung der Endungsvokale im Infinitiv, also $\langle-\mathrm{u}\rangle$ und seltener $\langle-\mathrm{o}\rangle$ im Chatraum \#wallis, $\langle-\mathrm{a}\rangle$ im Chatraum \#graubuenden und $\langle-\mathrm{e}\rangle$ in den mittelländischen Chaträumen (\#bern und \#zuerich). Neben den Formen, die die lautliche Form wiedergeben, sind aber auch gewisse Normalformen zu finden, insbesondere 〈-e unbetonte Vokale in den Alpinmundartchats. In den mittelländischen Chaträumen erscheint für das Endungsschwa häufig eine Graphie <-ä〉, womit die lautliche Differenz zum standardsprachlichen Schwa markiert wird.

Das zweite Beispiel dokumentiert, dass die im Mittelland weitgehend geschwunden t-Flexive bei der 2. und 3. Person Singular von "sein" in den Alpinmundarten noch immer vorkommen. Zudem findet sich eine bemerkenswerte Verteilung, dass nämlich das t-Flexiv im Wallis die 2. Person markiert, während es in Graubünden für die 3. Person verwendet wird. Für eine solche Verteilung findet sich im SDS (noch) keine Grundlage. Neben dem generellen Schwund zeigen die umfangreichen Daten also eine neue Funktionalisierung alter Formen. Dies kann als erster Schritt für Sprachwandel interpretiert werden, falls sich die Formen in dieser Verteilung durchsetzen können. Das Ergebnis zeigt zudem, dass im Chat nicht nur Neologismen von Bedeutung sind, sondern dass auch archaische Formen in modernsten Kommunikationsformen unter mehrheitlich jungen Usern weiterleben.

Die Analyse der Verschriftung von Neologismen zeigt in der Morphologie die traditionelle sprachgeographische Verteilung, die Untersuchung der restlichen Schreibung ist weniger ergiebig. Sie dokumentiert jedoch die Gültigkeit der Rechtschreibeprinzipien über die Standardsprache hinaus.

Insgesamt konnte dieser Aufsatz auch aufzeigen, dass die Mundartverschriftung in Schweizer Chats für die Dialektologie fruchtbar gemacht werden kann. Ein umfangreiches Korpus kann ohne großen Aufwand erhoben werden und ist, weil es schon in verschrifteter Form vorliegt, einer Analyse unmittelbar zugänglich. Die Laienverschriftung bietet zwar eine Reduktion der phonetischen Exaktheit, für Analysen der Morphologie und die hier nicht untersuchten Bereiche Syntax und Lexikon sind die Daten aber tauglich. Auch für lautliche Aspekte können verschriftete Beiträge mit Einschränkungen genutzt werden, wie das in der deutschen Dialektologie seit Wenkers Sprachatlanten deutlich wird und wie es hier mit der Untersuchung der Infinitivendung gezeigt wurde. Was ein Chat-Korpus nicht bieten kann, ist eine sichere Lokalisierung, da die ChatterInnen anonym sind. Der areale Aspekt, mithin die wichtigste außersprachliche Größe der Dialektologie, ist insofern nur beschränkt gültig. Die hier dargestellten Analysen zeigen aber, dass diese Einschränkung durch die große Datenmenge zumindest teilweise wettgemacht wird. Die Analyse von mundartlichen Chats bietet somit auch für die Dialektologie neue Möglichkeiten. 


\section{Literaturangaben}

Aschwanden, Brigitte (2001): "Wär wot chätä?". Zum Sprachverhalten deutschschweizerischer Chatter. Online (Networx 24).

http://www.websprache.net/networx/docs/networx-24.pdf

Christen, Helen (1998): Dialekt im Alltag. Eine empirische Untersuchung zur lokalen Komponente heutiger schweizerdeutscher Varietäten. Tübingen. $(=R G L 201)$.

Christen, Helen/Tophinke, Doris/Ziegler, Evelyn (i. Dr.): "Chat und regionale Identität". In: Wolf, Norbert Richard (ed.): Akten der Konferenz "Bayerische Dialektologie", Würzburg 26.-28.2.2002.

Christen Helen (eingereicht): "Dialekt-Schreiben oder sorry ech hassä Text schribä". In: Glaser, Elvira/Ott, Peter/Schwarzenbach, Ruedi (ed.): Tagungsband der 14. Arbeitstagung zur alemannischen Dialektologie in Männedorf/Zürich 16. - 18.9.2002.

Dieth, Eugen (1938): Schwyzertütschi Dialäktschrift. Leitfaden einer einheitlichen Schreibweise für alle Dialekte. Nach den Beschlüssen der Schriftkommission der Neuen Helvetischen Gesellschaft, Gruppe Zürich, ausgearbeitet. Zürich.

Kelle, Bernhard (2000): "Regionale Varietäten im Internet - Chats als Wegbereiter einer regionalen Schriftlichkeit?". Deutsche Sprache 27: 357-371.

Kelle, Bernhard (2002): "Virtual Dialect Areas in the Internet: 'Townchats'". In: Gaul, Wolfgang/Ritter, Gunter (eds.): Classification, Automation, and New Media. Berlin et al.: 281-288.

Koch, Peter/Oesterreicher, Wulf (1994): "Schriftlichkeit und Sprache". In: Günther, Hartmut/ Ludwig, Otto (eds.): Schrift und Schriftlichkeit. Writing and Its Use. Ein interdisziplinäres Handbuch internationaler Forschung. An Interdisciplinary Handbook of International Research. Berlin/New York: 587-604. (=Handbücher zur Sprach- und Kommunikationswissenschaft 10.1).

Lötscher, Andreas (1989): "Probleme und Problemlösungen bei der Mundartschreibung des Schweizerdeutschen". Zeitschrift für Dialektologie und Linguistik 56: 273-297.

Lötscher, Andreas (1990): "Zum Problem der Normalisierung der Mundartschreibung im Schweizerdeutschen". In: Marthe, Philipp (ed.): Alemannische Dialektologie im ComputerZeitalter. Göppingen: 191-207. (=Göppinger Arbeiten zur Germanistik 535).

Marti, Werner (1972): Bärndütschi Schrybwys. Ein Wegweiser zum Aufschreiben in berndeutscher Sprache, mit einer Einführung über allgemeine Probleme des Aufschreibens und einem Wörterverzeichnis nebst Beispielen. Bern.

Runkehl, Jens/Schlobinski, Peter/Siever, Torsten (1998): Sprache und Kommunikation im Internet. Überblick und Analysen. Opladen/Wiesbaden.

Schmid, Christian (2003): "'Bach- u Wöschtag'. Deutschschweizer Mundartliteratur am Ende des 20. Jahrhunderts". In: Dittli, Beat/Häcki Buhofer, Annelies/Haas, Walter (eds.): Gömmer MiGro? Veränderungen und Entwicklungen im heutigen SchweizerDeutschen. Freiburg: 193-204. (=Germanistica Friburgensia 18). 

und Verschriftung in schweizerdeutschen Chats

SDS: Sprachatlas der deutschen Schweiz (SDS). Begründet von Heinrich Baumgartner und Rudolf Hotzenköcherle. In Zusammenarbeit mit Konrad Lobeck, Robert Schläpfer, Rudolf Trüb und unter Mitwirkung von Paul Zinsli herausgegeben von Rudolf Hotzenköcherle. (1962-1997) Bern, Bd. VII und VIII Basel. 